\title{
Machine-learning and Automatically Segmented Retinal Biomarkers Generate Spatial Heatmaps Predictive for Standard and Low Luminance Visual Acuity in Geographic Atrophy
}

\section{Konstantinos Balaskas ( $\nabla$ k.balaskas@nhs.net)}

NIHR Biomedical Research Centre At Moorfields Eye Hospital NHS Foundation Trust, UCL Institute of Ophthalmology

\section{Sophie Glinton}

NIHR Biomedical Research Centre At Moorfields Eye Hospital NHS Foundation Trust, UCL Institute of Ophthalmology

Tiarnan Keenan

National Eye Institute, National Institutes of Health

\section{Livia Faes}

NIHR Biomedical Research Centre At Moorfields Eye Hospital NHS Foundation Trust, UCL Institute of Ophthalmology

\section{Bart Liefers}

Erasmus University Medical Center

\section{Gongyu Zhang}

NIHR Biomedical Research Centre At Moorfields Eye Hospital NHS Foundation Trust, UCL Institute of Ophthalmology

Nikolas Pontikos

NIHR Biomedical Research Centre At Moorfields Eye Hospital NHS Foundation Trust, UCL Institute of Ophthalmology

\section{Robbert Struyven}

NIHR Biomedical Research Centre At Moorfields Eye Hospital NHS Foundation Trust, UCL Institute of Ophthalmology

\section{Siegfried Wagner}

NIHR Biomedical Research Centre At Moorfields Eye Hospital NHS Foundation Trust, UCL Institute of Ophthalmology

\section{Alex McKeown}

Apellis Pharmaceuticals, Inc, Waltham, Massachusetts

\section{Praveen Patel}

NIHR Biomedical Research Centre At Moorfields Eye Hospital NHS Foundation Trust, UCL Institute of Ophthalmology 


\section{Pearse Keane}

NIHR Biomedical Research Centre At Moorfields Eye Hospital NHS Foundation Trust, UCL Institute of Ophthalmology

\section{Dun Jack Fu}

NIHR Biomedical Research Centre At Moorfields Eye Hospital NHS Foundation Trust, UCL Institute of Ophthalmology

\section{Research Article}

Keywords: biomarkers, machine learning, visual acuity, low luminance visual acuity, ophthalmology, geographic atrophy, qOCT

Posted Date: January 6th, 2022

DOl: https://doi.org/10.21203/rs.3.rs-1197225/v1

License: (c) (i) This work is licensed under a Creative Commons Attribution 4.0 International License. Read Full License 


\section{Abstract}

Objective: Predict visual function with machine-learning applied to automatically acquired quantitative imaging biomarkers in geographic atrophy

Design: Post-hoc analysis of data from a clinical trial and routine clinical care.

Methods: Automated segmentation of OCT scans from 476 eyes (325 patients) with geographic atrophy. Machine learning modelling of resultant quantitative OCT (qOCT) biomarkers to predict cross-sectional visual acuity under both standard luminance (VA) and low luminance (LLVA) conditions.

Main Outcome Measure: Correlation coefficient $\left(\mathrm{R}^{2}\right)$ and mean absolute error (MAE) for cross-sectional VA and LLVA in Early Treatment Diabetic Retinopathy Study (ETDRS) letters.

Results: Best-corrected VA under both standard luminance ( $R^{2}$ 0.46 MAE 10.2 ETDRS letters) and lowluminance conditions ( $R^{2} 0.25$ MAE 12.1) could be predicted. The foveal region contributed the most $(46.5 \%)$ toward model performance, with retinal pigment epithelium loss and outer retinal atrophy contributing the most (31.1\%). For LLVA, however, features in the non-foveal regions were most important (74.5\%), led by photoreceptor degeneration (38.9\%).

Conclusions: Our method of automatic qOCT segmentation demonstrates functional significance for vision in geographic atrophy, including LLVA. LLVA is itself predictive of geographic atrophy progression, implying that the predictive qOCT biomarkers provided by our model are also prognostic.

\section{Introduction}

Geographic atrophy (GA) is a chronic progressive degeneration of the macula, the $24 \mathrm{~mm}^{2}$ region of the retina required for central vision. It is the defining lesion of late age-related macular degeneration (AMD). (Schmitz-Valckenberg et al. 2016; Gass 1973) GA is associated with significant, irreversible vision loss. To assess visual function in AMD, visual acuity (VA) measured using a eye chart under standardised lighting is an established key outcome measure as defined by the International Consortium for Health Outcomes Measurement.(Rodrigues et al. 2016) Interestingly, in early stages of AMD, VA measured under low luminance conditions (low luminance visual acuity [LLVA]) can be reduced whilst standard VA remains unaffected. Most patients initially present with non-central GA (affecting the parafoveal region of the macula) and gradually proceed to foveal involvement (central GA).(Danis, Lavine, and Domalpally 2015; Keenan et al. 2018; Sunness 2008) In this context, LLVA correlates with future deterioration of VA and its reduction indicates that the patient will head towards the beginning of end-stage disease, and loss of foveal function may be impending.(Sunness et al. 2008; Wood et al. 2021) Yet the physiological mechanism underlying this outcome measure is unknown.(Stockman and Sharpe 2006; Zele and Cao 2014) 
With no current treatments for GA and promising therapies on the horizon,(Liao et al. 2020; Kuppermann et al. 2021; Allingham, Mettu, and Cousins 2019) it is increasingly important to establish structurefunction relationships within GA, as they: (i) provide further understanding of the pathophysiological mechanisms underlying $\mathrm{GA}$; (ii) refine monitoring of disease activity and thereby diagnostic and prognostic counselling at the individual patient level; (iii) serve to define clinical endpoints in clinical trials and enable identification of earlier stages, i.e. opportunities for interventions that can prevent vision loss.

The current reference standard for diagnosing, characterising, and monitoring progression in GA is spectral domain optical coherence tomography (SD-OCT), as it captures the cross-sectional morphology of retinal structures.(Holz et al. 2017) Indeed, an international consortium of experts in AMD and retinal imaging - the Consensus of Atrophy Meetings (CAM) group - chose to define disease progression in GA based on SD-OCT structural markers.(Sadda et al. 2018; Schmitz-Valckenberg et al. 2016) Their proposed terms for macular atrophy in the context of AMD each describe the affected anatomical layers and representing distinct disease stages. Herein, complete RPE (retinal pigment epithelium) and outer retinal atrophy (CRORA) represents the endpoint of atrophy - encompassing GA - and is defined by regions of: choroidal hypertransmission with diameter $\geq 250 \mu \mathrm{m}$; RPE attenuation or disruption with diameter $\geq 250$ $\mu \mathrm{m}$; overlying photoreceptor degeneration; and absence of RPE tear.(Sadda et al. 2018) Regions in which these features overlap but are less than $250 \mu \mathrm{m}$ are termed incomplete RPE and outer retinal atrophy (iRORA) - a precursor stage to cRORA.(Guymer et al. 2020) Recently, we developed a deep learning model to automatically segment RPE-loss, photoreceptor degeneration, and hypertransmission from OCT scans and shown through external validation to be comparable with human specialist efforts.(Zhang et al. 2021) Regions in which these features overlap represent RORA (RPE and outer retinal atrophy) and can be considered as a continuous variable that encompasses both cRORA and IRORA.

To date, the relationship between OCT structural features and visual function in GA have relied on manual segmentation and do not consider the biomarkers defined in the CAM consensus statement.(Pfau et al. 2020; Sayegh et al. 2017) This study makes use of an externally-validated algorithm that automatically segments these quantitative OCT (qOCT) biomarkers, applying it to non-neovascular AMD datasets ( $\mathrm{n}=$ 476) from both clinical trials and routine clinical care. When combined with machine learning, both standard and low luminance VA could be predicted to a level that has yet to be achieved. Furthermore, spatial localisation of these structure-function relationships were mapped onto the macula providing insight into the (otherwise unknown) underlying physiological mechanism of LLVA.

\section{Methods}

\section{Study design}

This is a non-interventional, post hoc analysis of patients with GA secondary to non-neovascular AMD. Reporting adhered to guidelines for observational studies put forth by the Strengthening the Reporting of Observational Studies in Epidemiology (STROBE) statement.(Gallo et al. 2011)

\section{Study cohort}


This study considered data from two sources: participants enrolled in the FILLY trial (NCT02503332) (Steinle and Hamdani 2019; Liao et al. 2020) and real-world data collected as part of routine clinical care for patients with GA at Moorfields Eye Hospital NHS Foundation Trust, London, United Kingdom.

The FILLY trial was a phase II, international, multicenter clinical trial assessing safety, tolerability, and evidence of activity of intravitreal pegcetacoplan in eyes with GA secondary to non-neovascular AMD with best-corrected visual acuity (BCVA) greater than 24 Early Treatment Diabetic Retinopathy Study (ETDRS) letters (Supplementary Methods 1 and Supplementary Figure 1). Here, only baseline trial data were considered, i.e. the timepoint prior to initiation of the intervention. The VA values represent BCVA testing using ETDRS charts by certified examiners after refraction. BCVA under low luminance conditions (low luminance visual acuity; LLVA) was measured as for BCVA but with a 2.0 log neutral density filter covering the eye. Low luminance deficit (LLD) was defined as the difference between BCVA and LLVA (i.e. BCVA - LLVA).

Patients from Moorfields Eye Hospital were included if all of the following criteria were met (Supplementary Figure 1): they attended a medical retina clinic between 01-January-2016 and 31January-2019; GA was included as a term in the clinic correspondence letter; Heidelberg OCT scans (greater than 25 b-scans per volume) were obtained from both eyes within 15 days of the appointment; absence of prior anti-VEGF therapy; and the OCT scans were confirmed to contain GA secondary to nonneovascular AMD (according to manual validation by a reading centre expert grader at the Moorfields Reading Centre). Here, VA (with habitual correction or pinhole) was measured as part of a clinical examination with an ETDRS chart. Pinhole VA was used if better than VA with habitual correction. For any eyes with multiple timepoints meeting the eligibility criteria, the earliest timepoint was selected.

For all patients, only a single macular OCT scan and a corresponding VA measurement were considered per eye. All OCT volumes were acquired using Heidelberg Spectralis OCT and HRA (Heidelberg Engineering, Heidelberg, Germany) having equal to or greater than 25 b-scans covering $6 \times 6 \times 2 \mathrm{~mm}^{3}$.

The study was conducted in compliance with the tenets of the Declaration of Helsinki and has approval from the Institutional Review Board (research reference: ROAD17/031, clinical audit reference:

CA17/MR/28; NIHR Biomedical Research Centre, Moorfields Eye Hospital NHS Foundation Trust, Institute of Ophthalmology, University College London). The requirement for informed consent was waived by the review/ethics committee as this is the standard for use of retrospective, de-identified data for research within the UK National Health Service (NHS).

\section{Image analysis workflow}

All OCT volumes were processed using a deep learning model that automatically segments each of the three following features: RPE-loss, photoreceptor degeneration (evidence of outer limiting membrane loss, ellipsoid zone loss, interdigitation zone loss, or outer nuclear layer thinning), and hypertransmission (Figure 1a).(Zhang et al. 2021) A fourth model that segments RORA was also used. RORA was defined as overlapping regions of RPE-loss, photoreceptor degeneration, and hypertransmission, i.e. any retinal areas 
with all three features present and therefore encompassing both iRORA and cRORA.(Sadda et al. 2018; Göbel et al. 2011)

The automatic segmentation process assigns a probability for each of the features to each voxel within an OCT volume. Voxel spatial localisation was interpolated in relation to the central fovea, thereby standardising locations and enabling comparison between patients (Figure 1b). Central foveal points were manually annotated by a reading centre expert grader at the Moorfields Reading Centre. The spatial localisation of feature probabilities was also considered by dividing the macula into each of the ETDRS regions, i.e. mean probability within each of the nine ETDRS regions (foveal, 4 parafoveal, and 4 perifoveal areas for the nasal, temporal, superior, and inferior regions) (Figure 1c). The area of each segmented feature in square milimetres $\left(\mathrm{mm}^{2}\right)$ was considered by applying optimised probability thresholds identified from the original model development and validation.(Zhang et al. 2021)

\section{Predicting visual acuity}

A random forest regression model was trained using the segmentation output (i.e. the raw probabilities at the voxel level for each feature (RPE-loss, photoreceptor degeneration, hypertransmission, and RORA)) as input variables to predict cross-sectional VA in ETDRS letters. In separate analyses, LLVA and LLD were each considered as the dependent variable. The goodness of model fit was evaluated by comparing the coefficient of determination $\left(\mathrm{R}^{2}\right)$ and mean absolute error (MAE) computed from 100-fold bootstrapped models trained on $80 \%$ of the bootstrap sample and evaluated on the other $20 \%$ split at the patient level. Feature importance was calculated by the method incorporated in the scikit-learn implementation of random forest regression, whereby features' rankings for their variance reduction capability are averaged over the ensemble. Output was multiplied by 100 to give percentage contribution towards model performance. Analyses were carried out with Python (version 3.6.9) and summarised metrics in the text are expressed as median with \pm interquartile range (IQR), unless otherwise specified, as the measurements were not normally distributed.

\section{Results}

\section{Cohort demographics}

The study cohort comprised 476 eyes with GA secondary to non-neovascular AMD from 325 patients that were undergoing a clinical trial of pegcetacoplan in GA secondary to AMD $(n=195)$ or routine clinical care at a large UK tertiary centre $(n=130)$ (Supplementary Figure 1$)$. Overall median age was $80.5 \pm I Q R$ 11.1 years and the majority were female $(196 ; 60.3 \%)$ (Table 1$)$. 
Table 1

Cohort demographics.

\begin{tabular}{|llll|}
\hline & $\begin{array}{l}\text { Overall } \\
(\mathbf{n = 3 2 5 )}\end{array}$ & $\begin{array}{l}\text { FILLY } \\
(\mathbf{n = 1 9 5 )}\end{array}$ & $\begin{array}{l}\text { MEH } \\
(\mathbf{n = 1 3 0 )}\end{array}$ \\
\hline Gender & & & $73(56.2 \%)$ \\
\hline Female & $196(60.3 \%)$ & $123(63.1 \%)$ & $57(43.8 \%)$ \\
\hline Male & $129(39.7 \%)$ & $72(36.9 \%)$ & \\
\hline Age (years) & & & $79.1(9.12)$ \\
\hline Mean (SD) & $79.7(8.21)$ & $80.1(7.55)$ & $79.8[45.9,103]$ \\
\hline Median [Min, Max] & $80.5[45.9,103]$ & $80.7[60.4,97.2]$ & $1(0.8 \%)$ \\
\hline Ethnicity & & & $6(4.6 \%)$ \\
\hline Afro Caribbean & - & $1(0.5 \%)$ & $62(47.7 \%)$ \\
\hline Asian & - & - & $0(0 \%)$ \\
\hline Caucasian & - & $187(95.9 \%)$ & $61(46.9 \%)$ \\
\hline Other & - & $2(1.0 \%)$ & - \\
\hline Unknown & - & $0(0 \%)$ & $5(2.6 \%)$ \\
\hline Hispanic or Latino & - & &
\end{tabular}

Mean, median, minimum value, maximum value, standard deviation (SD), and proportional distributions are shown for gender, ethnicity, and age of cohort including patients with geographic atrophy secondary to non-neovascular AMD. Patients were recruited as part of an international, multicentre trial (FILLY) or retrospectively through the real-world clinical care at Moorfields Eye Hospital (MEH). Direct comparison of ethnicity was infeasible as each cohort represented ethnicities with distinct categorical variables that could not be intuitively merged.

\section{Clinical features and GA segmentation}

A wide distribution in visual function metrics was observed in the study cohort: median standard VA 60.5 \pm IQR 32.0 ETDRS letters, LLVA $32.0 \pm 28.0$, and LLD $21.0 \pm 21.0$ (Table 2 and Supplementary Figure 2a). The total area occupied by each feature was highly variable in the cohort, resulting in a distribution with median values of: $7.82 \pm 7.58 \mathrm{~mm}^{2}$ RPE-loss, $14.4 \pm 9.57 \mathrm{~mm}^{2}$ photoreceptor degeneration, $9.23 \pm 7.58$ $\mathrm{mm}^{2}$ hypertransmission, and $6.22 \pm 6.49 \mathrm{~mm}^{2}$ RORA (Table 2 and Supplementary Figure $2 \mathrm{~b}$ ). Presence of RORA and its constituent features was also highly variable within each region, ranging from completely absent $(0 \%)$ to confluent (>99\%). Indeed, RORA was wholly absent from the circular ETDRS foveal region (diameter $1 \mathrm{~mm} ; 0.79 \mathrm{~mm}^{2}$ ) in $14.7 \%$ of OCT volumes (70/476) (Table 2). 
Table 2. Visual function and corresponding GA feature segmentation.

\begin{tabular}{|llll|}
\hline (a) & $\begin{array}{l}\text { Overall } \\
(\mathrm{N}=476)\end{array}$ & $\begin{array}{l}\text { FILLY } \\
(\mathbf{N}=299)\end{array}$ & $\begin{array}{l}\text { MEH } \\
(\mathrm{N}=177)\end{array}$ \\
\hline Patients & 325 & 195 & 130 \\
\hline $\begin{array}{l}\text { Standard visual acuity } \\
\text { (ETDRS letters) }\end{array}$ & & & \\
\hline Mean (SD) & $55.1(21.1)$ & $57.5(18.5)$ & $51.2(24.4)$ \\
\hline Median [Min, Max] & $60.5[0,90.0]$ & $60.0[0,90.0]$ & $61.0[0,89.0]$ \\
\hline Low-luminance visual acuity & & & \\
\hline Mean (SD) & $33.3(17.5)$ & $33.3(17.5)$ & - \\
\hline Median [Min, Max] & $32.0[0,80.0]$ & $32.0[0,80.0]$ & - \\
\hline Missing & $179(37.6 \%)$ & $2(0.7 \%)$ & $177(100 \%)$ \\
\hline Low-luminance deficit & & & - \\
\hline Mean (SD) & $24.0(16.2)$ & $24.0(16.2)$ & - \\
\hline Median [Min, Max] & $21.0[0.00,73.0]$ & $21.0[0.00,73.0]$ & - \\
\hline Missing & $179(37.6 \%)$ & $2(0.7 \%)$ & $177(100 \%)$ \\
\hline
\end{tabular}




\begin{tabular}{|llll|}
\hline (b) & $\begin{array}{l}\text { Overall } \\
(\mathbf{N}=476)\end{array}$ & $\begin{array}{l}\text { FILLY } \\
(\mathbf{N}=299)\end{array}$ & $\begin{array}{l}\text { MEH } \\
\mathbf{( N = 1 7 7 )}\end{array}$ \\
\hline Patients & 325 & 195 & 130 \\
\hline RPE-loss $\left(\mathrm{mm}^{2}\right)$ & & & \\
\hline Mean (SD) & $8.53(5.18)$ & $8.21(4.29)$ & $9.06(6.37)$ \\
\hline Median [Min, Max] & $7.82[0.0309,25.0]$ & $7.49[0.603,20.8]$ & $8.17[0.0309,25.0]$ \\
\hline Photoreceptor degeneration $\left(\mathrm{mm}^{2}\right)$ & & & \\
\hline Mean (SD) & $14.2(6.38)$ & $13.8(5.60)$ & $14.7(7.49)$ \\
\hline Median [Min, Max] & $14.4[0.493,28.1]$ & $13.4[3.12,28.1]$ & $16.0[0.493,28.0]$ \\
\hline Hypertransmission $\left(\mathrm{mm}^{2}\right)$ & & & $10.2(6.56)$ \\
\hline Mean (SD) & & $9.44(4.50)$ & $9.59[0.227,24.8]$ \\
\hline Median [Min, Max] & $9.70(5.36)$ & $8.79[1.23,22.0]$ & \\
\hline RoRA (mm $\left.{ }^{2}\right)$ & $9.23[0.227,24.8]$ & & $7.79(5.97)$ \\
\hline Mean (SD) & & $6.91(3.87)$ & $6.56[0.0806,24.0]$ \\
\hline Median [Min, Max] & $7.24(4.77)$ & $6.03[0.584,17.5]$ & $6.22[0.0806,24.0]$ \\
\hline
\end{tabular}




\begin{tabular}{|llll|}
\hline (c) & $\begin{array}{l}\text { Overall } \\
(\mathbf{N}=476)\end{array}$ & $\begin{array}{l}\text { FILLY } \\
\mathbf{( N = 2 9 9 )}\end{array}$ & $\begin{array}{l}\text { MEH } \\
(\mathbf{N}=177)\end{array}$ \\
\hline Patients & 325 & 195 & 130 \\
\hline RPE-loss (\%) & & & \\
\hline Mean (SD) & $61.1(38.2)$ & $62.4(38.2)$ & $58.9(38.2)$ \\
\hline Median [Min, Max] & $77.0[0,99.0]$ & $80.0[0,99.0]$ & $73.0[0,99.0]$ \\
\hline Photoreceptor degeneration (\%) & & & \\
\hline Mean (SD) & $85.5(26.5)$ & $86.7(25.8)$ & $83.5(27.6)$ \\
\hline Median [Min, Max] & $99.0[0,99.0]$ & $99.0[0,99.0]$ & $99.0[0,99.0]$ \\
\hline Hypertransmission (\%) & & & \\
\hline Mean (SD) & $67.5(34.8)$ & $69.0(34.0)$ & $65.0(36.0)$ \\
\hline Median [Min, Max] & $83.0[0,99.0]$ & $84.0[0,99.0]$ & $80.0[0,99.0]$ \\
\hline RORA (\%) & & & \\
\hline Mean (SD) & $53.6(38.4)$ & $54.3(38.8)$ & $52.5(37.7)$ \\
\hline Median [Min, Max] & $62.0[0,99.0]$ & $62.0[0,99.0]$ & $60.0[0,99.0]$ \\
\hline
\end{tabular}

Mean, standard deviation (SD), and distribution are shown for (a) visual function metrics (standard visual acuity [VA] in ETDRS [early treatment diabetic retinopathy study] letters], low-luminance visual acuity [LLVA], low-luminance deficit [difference between VA and LLVA]); (b) feature segmentations (total areas in square millimetres [ $\left.\mathrm{mm}^{2}\right]$ of RPE-loss, photoreceptor degeneration, hypertransmission, and geographic atrophy; (c) proportion of each feature overlapping with central foveal region $\left(0.79 \mathrm{~mm}^{2}\right)$. Cohort data was collectively summarised (Overall) and sub-stratified by recruitment (FILLY trial or MEH [Moorfields Eye Hospital]). LLVA and LLD were not measured as part of routine clinical care at MEH and these metrics were not available for 2 persons of the FILLY sub-cohort - these were collectively referred to as missing.

\section{Prediction of standard visual acuity using machine learning}

For standard VA, the accuracy of using automatically segmented feature probabilities at the voxel level to predict VA had $R^{2} 0.40$, with mean absolute error (MAE) of 11.7 ETDRS letters (Table 3a). Of note, these features were more predictive of BCVA ( $R^{2} 0.46$ MAE 10.2) in the FILLY cohort than of best recorded VA measured as part of clinical examination ( $R^{2} 0.30 \mathrm{MAE} 15.3$ ) in the MEH cohort. Predictive importance for 
each feature and its position was ranked, wherein RORA contributed the most (31.3\%), followed by RPEloss (23.7\%), hypertransmission (25.4\%), and photoreceptor degeneration (19.6\%) (Figure 2a and Table $3 b)$. Feature position was further considered by summing feature importance across ETDRS regions. Critically, features within the foveal region contributed the most (46.5\%) despite being the smallest ETDRS region $-0.78 \mathrm{~mm}^{2}$ (Table $3 \mathrm{~b}$ ).

Table 3. Structure-function correlation between qOCT biomarkers of GA area and VA.

\begin{tabular}{|lll|}
\hline (a) & $\mathbf{R}^{2}$ & $\begin{array}{l}\text { MAE } \\
(25 \% \text { quartile }-75 \% \text { quartile })\end{array}$ \\
\hline Standard visual acuity & & \\
\hline Overall & 0.40 & $11.7(11.2-12.4)$ \\
\hline FILLY & 0.46 & $10.2(9.5-10.7)$ \\
\hline MEH & 0.30 & $15.3(13.8-16.6)$ \\
\hline Low-luminance visual acuity & & \\
\hline FILLY & 0.25 & $12.1(11.4-12.9)$ \\
\hline Low-luminance deficit & & \\
\hline FILLY & 0.25 & $10.1(9.5-10.8)$ \\
\hline
\end{tabular}


(b) Standard visual acuity

\begin{tabular}{|lllllll|}
\hline Region & $\begin{array}{l}\text { RPE- } \\
\text { loss }\end{array}$ & $\begin{array}{l}\text { Photoreceptor } \\
\text { degeneration }\end{array}$ & Hypertransmission & RORA & Sum \\
\hline 1 & Foveal & 13.4 & 6.8 & 9.5 & 16.8 & $\mathbf{4 6 . 5}$ \\
\hline 2 & Inner Superior & 2.3 & 1.4 & 2.2 & 2.7 & $\mathbf{8 . 5}$ \\
\hline 3 & Inner Nasal & 1.7 & 1.5 & 1.9 & 2.2 & $\mathbf{7 . 3}$ \\
\hline 4 & Inner Inferior & 1.7 & 1.5 & 1.9 & 2.0 & $\mathbf{7 . 1}$ \\
\hline 5 & $\begin{array}{l}\text { Inner } \\
\text { Temporal }\end{array}$ & 1.5 & 1.6 & 1.8 & 2.1 & $\mathbf{7 . 0}$ \\
\hline 6 & Outer Superior & 0.8 & 1.7 & 2.0 & 1.3 & $\mathbf{5 . 9}$ \\
\hline 7 & Outer Nasal & 0.9 & 1.9 & 2.1 & 1.6 & $\mathbf{6 . 5}$ \\
\hline 8 & Outer Inferior & 0.7 & 1.9 & 2.2 & 1.4 & $\mathbf{6 . 1}$ \\
\hline 9 & $\begin{array}{l}\text { Outer } \\
\text { Temporal }\end{array}$ & 0.6 & 1.4 & 1.9 & 1.2 & $\mathbf{5 . 1}$ \\
\hline & Sum & $\mathbf{2 3 . 7}$ & $\mathbf{1 9 . 6}$ & $\mathbf{2 5 . 4}$ & $\mathbf{3 1 . 3}$ & \\
\hline
\end{tabular}

(c) Low luminance visual acuity

\begin{tabular}{|lllllll|}
\hline Region & $\begin{array}{l}\text { RPE- } \\
\text { loss }\end{array}$ & $\begin{array}{l}\text { Photoreceptor } \\
\text { degeneration }\end{array}$ & Hypertransmission & RORA & Sum \\
\hline 1 & Foveal & 3.0 & 12.2 & 5.6 & 4.8 & $\mathbf{2 5 . 5}$ \\
\hline 2 & Inner Superior & 2.8 & 4.0 & 2.9 & 3.0 & $\mathbf{1 2 . 7}$ \\
\hline 3 & Inner Nasal & 1.7 & 3.1 & 2.2 & 2.2 & $\mathbf{9 . 2}$ \\
\hline 4 & Inner Inferior & 1.9 & 4.1 & 2.7 & 2.9 & $\mathbf{1 1 . 6}$ \\
\hline 5 & $\begin{array}{l}\text { Inner } \\
\text { Temporal }\end{array}$ & 1.6 & 3.3 & 2.3 & 2.4 & $\mathbf{9 . 5}$ \\
\hline 6 & Outer Superior & 0.9 & 3.7 & 2.7 & 1.7 & $\mathbf{8 . 9}$ \\
\hline 7 & Outer Nasal & 0.9 & 3.0 & 2.5 & 1.7 & $\mathbf{8 . 1}$ \\
\hline 8 & Outer Inferior & 0.5 & 3.0 & 2.5 & 1.3 & $\mathbf{7 . 3}$ \\
\hline 9 & $\begin{array}{l}\text { Outer } \\
\text { Temporal }\end{array}$ & 0.7 & 2.5 & 2.5 & 1.5 & $\mathbf{7 . 1}$ \\
\hline & Sum & $\mathbf{1 3 . 8}$ & $\mathbf{3 8 . 9}$ & $\mathbf{2 6 . 0}$ & $\mathbf{2 1 . 4}$ & \\
\hline
\end{tabular}


(a) A random forest regression model was trained using the segmentation output (i.e. the raw probabilities at the voxel level for each feature (RPE-loss, photoreceptor degeneration, hypertransmission, and RORA) as input variables to predict cross-sectional VA under standard luminance conditions, lowluminance VA, and low-luminance deficit in ETDRS letters. Model bootstrapped 100-fold with resultant Correlation coefficients $\left(\mathrm{R}^{2}\right)$ and mean absolute error (MAE) shown. Importance of qOCT biomarker features in predicting (b) standard visual acuity and (c) low luminance visual acuity was queried using machine learning. Random forests modelling was used to evaluate value of the qOCT biomarkers RPEloss, photoreceptor degeneration, hypertransmission, and RORA in predicting cross-sectional visual acuity under standard lighting conditions. The resultant adjusted feature importance values were summed according to location within ETDRS region and multiplied by 100 to give the percentage contribution towards the model's performance. For example, RORA within the foveal region accounted for $16.8 \%$ of the model's performance of $\mathrm{R}^{2}$ 0.40 MAE 11.7 ETDRS letters for standard visual acuity.

\section{Prediction of low luminance visual acuity using machine learning}

Additional visual parameters were available in the FILLY cohort. Here, cross-sectional correlation between model output feature probabilities and LLVA and LLD was assessed. $R^{2}$ of 0.25 (MAE 12.1) and 0.25 (MAE 10.1) was observed for LLVA and LLD, respectively (Table 3a). Ranking of feature importance for LLVA was led by photoreceptor degeneration (38.9\%), followed by hypertransmission (26.0\%), RORA (21.4\%), and RPE-loss (13.8\%) (Figure $2 \mathrm{~b}$ and Table $3 \mathrm{c}$ ). In contrast to standard VA, where feature importance was greatest for the foveal region, features at non-foveal regions were most important in predicting LLVA. When the VA and LLVA correlation models were repeated with one eye per patient, a similar pattern of feature importance was observed (Supplementary Table 1).

\section{Discussion}

\section{Main findings}

Our data demonstrate that both standard and low luminance VA in patients with GA secondary to nonneovascular AMD can be predicted using qOCT features that have been automatically segmented and quantified. This is the first time a structure-function relationship has been described for LLVA, creating a tool to assess its underlying physiological mechanisms. The predictive performance demonstrated for standard BCVA $\left(R^{2}=0.46\right.$; Table $\left.3 a\right)$ is superior to that from similar efforts in neovascular AMD also using machine-learning based segmentation algorithms $-R^{2}=0.11$ to 0.21. (Fu et al. 2021; SchmidtErfurth et al. 2018) An MAE of 10.9 ETDRS letters is a step towards the limit of predicting VA, as the testretest repeatability for standard VA is 5-6 ETDRS letters in eyes without disease and thought to be even greater in eyes with GA.(Siderov and Tiu 1999; Patel et al. 2008)

\section{Mapping structure and location using probability heatmaps}


The algorithm described here can produce a "GA feature probability heatmap", wherein the algorithm output of segmentation probabilities is interpolated in relation to the fovea and projected onto an en face fundus image. Probability maps allow us to instantly consider quantitative segmentation data across an entire image volume simultaneously through an intuitive graphic. Consideration of segmentation features as continuous variables (i.e. probability of feature presence) rather than binary variables (feature present or not present) obviates difficulties of assigning fixed thresholds. Landmarking each voxel to a reference point enables comparison of features over time and even between samples and patients (an obligate step for modelling).

\section{Structure-function correlation of standard visual acuity}

A stratified feature-region analysis suggested that foveal RORA, followed by foveal RPE-loss, is the strongest predictor of standard VA in GA. The strong foveal contribution to predicting standard VA is largely unsurprising, reflecting the clinical observation that central GA is accompanied by poor VA. (Keenan et al. 2018) Indeed, topographical analyses of fundus autofluorescence as well as SD-OCT have demonstrated that foveal sparing is an independent covariate of VA(Bagheri et al. 2019) and that VA is likely to be worse in eyes with definitive foveal involvement.(Lindner et al. 2017) To date, a correlation between total GA area and VA has not been readily apparent using fundus photography.(You et al. 2020; Lindner et al. 2017; Heier et al. 2020; Bagheri et al. 2019; Liefers et al. 2020) Here, we present evidence to show SD-OCT imaging can detect correlations between non-foveal features and VA. This may be because fundus photography only provides two-dimensional, en face representations of the retina, limiting indepth assessment of the retinal layers and discrimination between histological subtypes of GA and their respective contribution to functional deficit.(Liefers et al. 2021) Macular segmentation with SD-OCT thus presents a potentially more sensitive modality for GA and its sequelae on visual function.

Currently, GA is usually considered present when the lateral spread of RORA affects an area of atrophy $\geq$ $250 \mu \mathrm{m}$ in diameter.(Sadda et al. 2018) Using the algorithm described here allows evaluation of RORA as a continuous variable, and thresholding based on the extent of the lateral spread may be applied as a secondary step. This may facilitate the continuous monitoring and evaluation of GA in a research context, with a view to eventually develop clinical monitoring and preventative strategies.

\section{Predicting low luminance visual acuity}

LLVA is a simple, inexpensive, quick assessment with standard ophthalmic equipment and has a testretest repeatability (between 0.1 and 0.13 logMAR [5-6.5 ETDRS letters]) comparable to standard luminance VA.(Lovie-Kitchin and Brown 2000; Pluháček and Siderov 2018) It has been shown to correlate with microperimetry retinal sensitivities (Wu et al. 2015) and patient-reported night vision symptoms.(Wu, Guymer, and Finger 2016) LLVA is also an earlier clinical marker of change in central retinal function than standard VA. LLVA deterioration precedes deterioration in standard VA and thus predicts impending loss of foveal function. This is consistent with the structure-function correlation analyses presented here, as the most predictive features differed between the two measures of visual function: photoreceptor degeneration for LLVA and RPE-loss and RORA for standard VA. This suggests that photoreceptor 
degeneration can precede RPE-loss and eventual RORA in GA and aligns with current understanding that RPE dysfunction is common to all, or at least most, early AMD.(A. Bird 2020; A. C. Bird, Phillips, and Hageman 2014) That is, photoreceptor cells are metabolically dependent on RPE and therefore degeneration arises secondarily to RPE dysfunction, which itself eventually atrophies. Furthermore, features within non-foveal areas were more predictive of LLVA than for standard VA. This might support the hypothesis that low light sensitivity is mediated by a circuit function of horizontal and amacrine cells within the plexiform layers and thus a larger area of preserved central macula is required for LLVA. (Owsley et al. 2016)

\section{Limitations}

VA is the most commonly used functional measure to evaluate the visual system. It is widely accepted in clinics and by regulatory authorities as a key measure of visual function and represents the gold standard by which the efficacy of treatment is judged. It correlates with quality of life and defines key functional thresholds, such as eligibility for driving and for sight impairment registration. However, VA change over time is non-linear, can improve from one timepoint to the next, and does not wholly capture the sequelae of GA on visual function, as it largely represents central acuity of the fovea.(Csaky et al. 2017) Other functional manifestations include parafoveal functions such as dark adaptation, reading speed, face recognition, and perimetry. Foveal-sparing disease (i.e. not affecting VA) can impact other visual functions (including reading speed, contrast sensitivity, fixation, and VFQ-25).(Burguera-Giménez et al. 2020) Changes in visual function can even occur prior to VA deterioration. Thus, other markers of visionrelated performance in everyday life should be considered to complement VA in patients with GA.

\section{Conclusion}

Our results demonstrate the utility of automatically segmented imaging biomarkers in predicting visual outcomes. This is an important step towards standardising care and may contribute to the development of point-to-care decision-aid systems for personalised ophthalmology. Here we have used this tool to further our insight into the (otherwise unknown) underlying physiological mechanism of LLVA and thereby progression from early AMD to GA.

\section{Declarations}

Funding/support: Apellis Pharmaceuticals (Waltham, Massachusetts, United States) provided financial support and critical review of manuscript for publication.

\section{Author contributions statements}

KB, PAK, DJF: research design. KB, SG, TK, LF, BL, GZ, SW, PK, DJF: Data analysis, interpretation, research execution. All authors contributed towards the preparation of the manuscript and approved the final submitted version. Corresponding author is solely responsible for managing communication between co- 
authors; that all authors are included in the author list; order has been agreed by all authors; and that all authors are aware that the paper was submitted.

\section{Financial disclosures and conflict of interest:}

NP: Moorfields Eye Charity Career Development Award (R190031A), equity owner, Phenopolis Ltd.

DJF: Consulting for Abbvie, Allergan, DeepMind

LF: Nothing to declare

GZ: No conflicts of interest

BL: No conflicts of interest.

SG: Moorfields Eye Charity Grant (GR001003), Wellcome Trust Grant (206619_Z_17_Z).

SKW: MRC Clinical Research Training Fellowship (MR/T000953/1). RS: No conflicts of interest.

PAK: Moorfields Eye Charity Career Development Award (R190028A), UK Research \& Innovation Future Leaders Fellowship (MR/T019050/1); Consulting for DeepMind, Roche, Novartis, Apellis and BitFount; equity owner in Big Picture Medical; speaker fees from Heidelberg Engineering, Topcon, Allergan, and Bayer.

KB: Speaker fees from Novartis, Bayer, Alimera, Allergan and Heidelberg, consulting Novartis and Roche and research support from Apellis, Novartis and Bayer.

PJP: Speaker fees from Bayer, Heidelberg, Roche and Topcon, consulting Bayer, Novartis, Oxford Bioelectronics and Roche and research support from Bayer.

AM: Employee of Apellis

TK: No conflicts of interest

\section{References}

1. Allingham, Michael J., Priyatham S. Mettu, and Scott W. Cousins. 2019. “Elamipretide, a Mitochondrial-Targeted Drug, for the Treatment of Vision Loss in Dry AMD with High Risk Drusen: Results of the Phase 1 ReCLAIM Study." Ethnicity 24: 60-60.

2. Bagheri, Saghar, Ines Lains, Rebecca F. Silverman, Ivana Kim, Dean Eliott, Rufino Silva, John Miller, et al. 2019. "Percentage of Foveal vs Total Macular Geographic Atrophy as a Predictor of Visual Acuity in Age-Related Macular Degeneration." Journal of Vitreoretinal Diseases 3 (5): 278-82.

3. Bird, Alan. 2020. "Role of Retinal Pigment Epithelium in Age-Related Macular Disease: A Systematic Review." The British Journal of Ophthalmology, September. https://doi.org/10.1136/bjophthalmol- 
2020-317447.

4. Bird, Alan C., Rachel L. Phillips, and Gregory S. Hageman. 2014. “Geographic Atrophy: A Histopathological Assessment." JAMA Ophthalmology 132 (3): 338-45.

5. Burguera-Giménez, Noemi, Santiago García-Lázaro, Enrique España-Gregori, Roberto Gallego-Pinazo, Neus Burguera-Giménez, Manuel Rodríguez-Vallejo, and Gowtham Jonna. 2020. "Multimodal Evaluation of Visual Function in Geographic Atrophy versus Normal Eyes." Clinical Ophthalmology 14 (June): 1533-45.

6. Csaky, Karl, Frederick Ferris 3rd, Emily Y. Chew, Prashant Nair, Janet K. Cheetham, and Jacque L. Duncan. 2017. "Report From the NEI/FDA Endpoints Workshop on Age-Related Macular Degeneration and Inherited Retinal Diseases." Investigative Ophthalmology \& Visual Science 58 (9): 3456-63.

7. Danis, Ronald P., Jeremy A. Lavine, and Amitha Domalpally. 2015. "Geographic Atrophy in Patients with Advanced Dry Age-Related Macular Degeneration: Current Challenges and Future Prospects." Clinical Ophthalmology 9 (November): 2159-74.

8. Fu, Dun Jack, Livia Faes, Siegfried K. Wagner, Gabriella Moraes, Reena Chopra, Praveen J. Patel, Konstantinos Balaskas, Tiarnan D. L. Keenan, Lucas M. Bachmann, and Pearse A. Keane. 2021. "Predicting Incremental and Future Visual Change in Neovascular Age-Related Macular Degeneration Using Deep Learning." Ophthalmology. Retina, January. https://doi.org/10.1016/j.oret.2021.01.009.

9. Gallo, Valentina, Matthias Egger, Valerie McCormack, Peter B. Farmer, John P. A. Ioannidis, Micheline Kirsch-Volders, Giuseppe Matullo, et al. 2011. "STrengthening the Reporting of OBservational Studies in Epidemiology - Molecular Epidemiology (STROBE-ME): An Extension of the STROBE Statement." Mutagenesis 27 (1): 17-29.

10. Gass, J. D. M. 1973. “Drusen and Disciform Macular Detachment and Degeneration.” Archives of Ophthalmology. https://doi.org/10.1001/archopht.1973.01000050208006.

11. Göbel, Arno P., Monika Fleckenstein, Steffen Schmitz-Valckenberg, Christian K. Brinkmann, and Frank G. Holz. 2011. “Imaging Geographic Atrophy in Age-Related Macular Degeneration.” Ophthalmologica. Journal International D'ophtalmologie. International Journal of Ophthalmology. Zeitschrift Fur Augenheilkunde 226 (4): 182-90.

12. Guymer, Robyn H., Philip J. Rosenfeld, Christine A. Curcio, Frank G. Holz, Giovanni Staurenghi, K. Bailey Freund, Steffen Schmitz-Valckenberg, et al. 2020. “Incomplete Retinal Pigment Epithelial and Outer Retinal Atrophy in Age-Related Macular Degeneration: Classification of Atrophy Meeting Report 4." Ophthalmology 127 (3): 394-409.

13. Heier, Jeffrey S., Dante Pieramici, Usha Chakravarthy, Sunil S. Patel, Sunil Gupta, Andrew Lotery, Eleonora M. Lad, et al. 2020. "Visual Function Decline Resulting from Geographic Atrophy: Results from the Chroma and Spectri Phase 3 Trials." Ophthalmology. Retina 4 (7): 673-88.

14. Holz, Frank G., Srinivas R. Sadda, Giovanni Staurenghi, Moritz Lindner, Alan C. Bird, Barbara A. Blodi, Ferdinando Bottoni, et al. 2017. "Imaging Protocols in Clinical Studies in Advanced Age-Related 
Macular Degeneration: Recommendations from Classification of Atrophy Consensus Meetings." Ophthalmology 124 (4): 464-78.

15. Keenan, Tiarnan D., Elvira Agrón, Amitha Domalpally, Traci E. Clemons, Freekje van Asten, Wai T. Wong, Ronald G. Danis, et al. 2018. "Progression of Geographic Atrophy in Age-Related Macular Degeneration: AREDS2 Report Number 16." Ophthalmology 125 (12): 1913-28.

16. Kuppermann, Baruch D., Sunil S. Patel, David S. Boyer, Albert J. Augustin, William R. Freeman, Kevin J. Kerr, Qiang Guo, Susan Schneider, Francisco J. López, and Brimo DDS Gen 1 Study Group. 2021. "PHASE 2 STUDY OF THE SAFETY AND EFFICACY OF BRIMONIDINE DRUG DELIVERY SYSTEM (BRIMO DDS) GENERATION 1 IN PATIENTS WITH GEOGRAPHIC ATROPHY SECONDARY TO AGERELATED MACULAR DEGENERATION." Retina 41 (1): 144-55.

17. Liao, David S., Federico V. Grossi, Delphine El Mehdi, Monica R. Gerber, David M. Brown, Jeffrey S. Heier, Charles C. Wykoff, et al. 2020. "Complement C3 Inhibitor Pegcetacoplan for Geographic Atrophy Secondary to Age-Related Macular Degeneration: A Randomized Phase 2 Trial." Ophthalmology 127 (2): 186-95.

18. Liefers, Bart, Johanna M. Colijn, Cristina González-Gonzalo, Timo Verzijden, Jie Jin Wang, Nichole Joachim, Paul Mitchell, et al. 2020. "A Deep Learning Model for Segmentation of Geographic Atrophy to Study Its Long-Term Natural History." Ophthalmology 127 (8): 1086-96.

19. Liefers, Bart, Paul Taylor, Abdulrahman Alsaedi, Clare Bailey, Konstantinos Balaskas, Narendra Dhingra, Catherine A. Egan, et al. 2021. "Quantification of Key Retinal Features in Early and Late AgeRelated Macular Degeneration Using Deep Learning." American Journal of Ophthalmology 226 (June): 1-12.

20. Lindner, Moritz, Jennifer Nadal, Matthias M. Mauschitz, Anna Lüning, Joanna Czauderna, Maximilian Pfau, Steffen Schmitz-Valckenberg, Frank G. Holz, Matthias Schmid, and Monika Fleckenstein. 2017. "Combined Fundus Autofluorescence and Near Infrared Reflectance as Prognostic Biomarkers for Visual Acuity in Foveal-Sparing Geographic Atrophy." Investigative Ophthalmology \& Visual Science 58 (6): BIO61-67.

21. Lovie-Kitchin, Jan E., and Brian Brown. 2000. "Repeatability and Intercorrelations of Standard Vision Tests as a Function of Age." Optometry and Vision Science. https://doi.org/10.1097/00006324200008000-00008.

22. Owsley, Cynthia, Mark E. Clark, Carrie E. Huisingh, Christine A. Curcio, and Gerald McGwin. 2016. "Visual Function in Older Eyes in Normal Macular Health: Association with Incident Early Age-Related Macular Degeneration 3 Years Later." Investigative Opthalmology \& Visual Science. https://doi.org/10.1167/iovs.15-18962.

23. Patel, Praveen J., Fred K. Chen, Gary S. Rubin, and Adnan Tufail. 2008. "Intersession Repeatability of Visual Acuity Scores in Age-Related Macular Degeneration." Investigative Ophthalmology \& Visual Science 49 (10): 4347-52.

24. Pfau, Maximilian, Leon von der Emde, Chantal Dysli, Philipp T. Möller, Sarah Thiele, Moritz Lindner, Matthias Schmid, et al. 2020. "Determinants of Cone and Rod Functions in Geographic Atrophy: Al- 
Based Structure-Function Correlation." American Journal of Ophthalmology 217 (September): 16273.

25. Pluháček, František, and John Siderov. 2018. “Mesopic Visual Acuity Is Less Crowded.” Graefe's Archive for Clinical and Experimental Ophthalmology = Albrecht von Graefes Archiv Fur Klinische Und Experimentelle Ophthalmologie 256 (9): 1739-46.

26. Rodrigues, Ian A., Sara M. Sprinkhuizen, Daniel Barthelmes, Mark Blumenkranz, Gemmy Cheung, Julia Haller, Robert Johnston, et al. 2016. “Defining a Minimum Set of Standardized Patient-Centered Outcome Measures for Macular Degeneration." American Journal of Ophthalmology 168 (August): 1-12.

27. Sadda, Srinivas R., Robyn Guymer, Frank G. Holz, Steffen Schmitz-Valckenberg, Christine A. Curcio, Alan C. Bird, Barbara A. Blodi, et al. 2018. "Consensus Definition for Atrophy Associated with AgeRelated Macular Degeneration on OCT: Classification of Atrophy Report 3." Ophthalmology 125 (4): 537-48.

28. Sayegh, Ramzi Gilbert, Stefan Sacu, Roman Dunavölgyi, Maria Elisabeth Kroh, Philipp Roberts, Christoph Mitsch, Alessio Montuoro, Margit Ehrenmüller, and Ursula Schmidt-Erfurth. 2017. “Geographic Atrophy and Foveal-Sparing Changes Related to Visual Acuity in Patients With Dry AgeRelated Macular Degeneration Over Time." American Journal of Ophthalmology 179 (July): 118-28.

29. Schmidt-Erfurth, Ursula, Hrvoje Bogunovic, Amir Sadeghipour, Thomas Schlegl, Georg Langs, Bianca S. Gerendas, Aaron Osborne, and Sebastian M. Waldstein. 2018. "Machine Learning to Analyze the Prognostic Value of Current Imaging Biomarkers in Neovascular Age-Related Macular Degeneration." Ophthalmology. Retina 2 (1): 24-30.

30. Schmitz-Valckenberg, Steffen, Srinivas Sadda, Giovanni Staurenghi, Emily Y. Chew, Monika Fleckenstein, Frank G. Holz, and CAM (Classification of Atrophy Meeting)-Group. 2016. “GEOGRAPHIC ATROPHY: Semantic Considerations and Literature Review.” Retina 36 (12): 225064.

31. Siderov, J., and A. L. Tiu. 1999. "Variability of Measurements of Visual Acuity in a Large Eye Clinic." Acta Ophthalmologica Scandinavica 77 (6): 673-76.

32. Steinle, Nathan, and Mohamed Hamdani. 2019. “Evaluation of Baseline Factors on Progression in a Large Phase-2 Clinical Trial for Geographic Atrophy (FILLY Study)." Investigative Ophthalmology \& Visual Science 60 (9): 973-973.

33. Stockman, Andrew, and Lindsay T. Sharpe. 2006. "Into the Twilight Zone: The Complexities of Mesopic Vision and Luminous Efficiency." Ophthalmic \& Physiological Optics: The Journal of the British College of Ophthalmic Opticians 26 (3): 225-39.

34. Sunness, Janet S. 2008. "Face Fields and Microperimetry for Estimating the Location of Fixation in Eyes with Macular Disease." Journal of Visual Impairment \& Blindness 102 (11): 679-89.

35. Sunness, Janet S., Gary S. Rubin, Aimee Broman, Carol A. Applegate, Neil M. Bressler, and Barbara S. Hawkins. 2008. "Low Luminance Visual Dysfunction as a Predictor of Subsequent Visual Acuity 
Loss from Geographic Atrophy in Age-Related Macular Degeneration." Ophthalmology 115 (9): 1480-88, 1488.e1-2.

36. Wood, Laura J., Jasleen K. Jolly, Thomas Mw Buckley, Amandeep S. Josan, and Robert E. MacLaren. 2021. "Low Luminance Visual Acuity as a Clinical Measure and Clinical Trial Outcome Measure: A Scoping Review." Ophthalmic \& Physiological Optics: The Journal of the British College of Ophthalmic Opticians 41 (2): 213-23.

37. Wu, Zhichao, Lauren N. Ayton, Chi D. Luu, and Robyn H. Guymer. 2015. "Longitudinal Changes in Microperimetry and Low Luminance Visual Acuity in Age-Related Macular Degeneration." JAMA Ophthalmology 133 (4): 442-48.

38. Wu, Zhichao, Robyn H. Guymer, and Robert P. Finger. 2016. "Low Luminance Deficit and Night Vision Symptoms in Intermediate Age-Related Macular Degeneration." The British Journal of Ophthalmology 100 (3): 395-98.

39. You, Jong In, Eung Suk Kim, Seung-Young Yu, Kiyoung Kim, and Others. 2020. "Correlation between Topographic Progression of Geographic Atrophy and Visual Acuity Changes."

https://www.researchsquare.com/article/rs-68760/latest.pdf.

40. Zele, Andrew J., and Dingcai Cao. 2014. "Vision under Mesopic and Scotopic Illumination." Frontiers in Psychology 5: 1594.

41. Zhang, Gongyu, Dun Jack Fu, Bart Liefers, Livia Faes, Sophie Glinton, Siegfried Wagner, Robbert Struyven, Nikolas Pontikos, Pearse A. Keane, and Konstantinos Balaskas. 2021. "Clinically Relevant Deep Learning for Detection and Quantification of Geographic Atrophy from Optical Coherence Tomography: A Model Development and External Validation Study." The Lancet Digital Health 3 (10): e665-75.

\section{Supplementary Figure}

Supplementary Figure 2 is not available with this version

\section{Figures}

\section{Image not available with this version}

\section{Figure 1}

\section{Image analysis workflow.}


(a) For each OCT volume, all b-scans were segmented for RPE-loss (orange), photoreceptor degeneration (blue), hypertransmission (red), and RPE and outer retinal atrophy (RORA; green). RORA is taken to be overlapping regions of the three former features i.e. co-occurrence as per a-scan. Exemplar segmentation of a single b-scan and its axis along en face fundus photograph. (b) Resultant feature probability maps from total volume segmentations collectively presented by projection onto en face fundus photograph. Colour legends represent target feature probability. Manual central foveal point annotation permitted interpolation of a given voxel's localisation in relation to the fovea. (c) ETDRS regions were also considered wherein the macula is considered as a $6 \mathrm{~mm}$ diameter circle divided into 9 areas: central foveal area ( $1 \mathrm{~mm}$ diameter); 4 parafoveal (collectively span $3 \mathrm{~mm}$ diameter); and 4 perifoveal areas. Here, the mean feature probability within each region is displayed.

\section{Image not available with this version}

\section{Figure 2}

\section{Heatmap of relative feature predictive value.}

Normalised random forest feature importance as a measure of the predictive value of the four considered features of GA; RPE-loss, photoreceptor degeneration, and hypertransmission and RORA and their locations relative to the fovea to the predicted value for (a) standard visual acuity and (b) low luminance visual acuity. Feature importance values were averaged across 100 bootstraps of the dataset.

\section{Supplementary Files}

This is a list of supplementary files associated with this preprint. Click to download.

- SupplementaryTable1.docx

- SupplementaryMaterials.docx

- supplementaryfigure1.pdf 